\title{
SIMULATION OF DENDRITE GROWTH OF CU-9AI ALLOY IN THE CONTINUOUS CASTING PROCESS
}

\author{
Robert PEZER ${ }^{1}$, Ivana IVANIĆ ${ }^{1}$, Stjepan KOŽUH ${ }^{1}$, Ivan ANŽEL ${ }^{2}$, Mirko GOJIĆ ${ }^{1}$ \\ ${ }^{1}$ University of Zagreb Faculty of Metallurgy, Sisak, Croatia, EU \\ rpezer@simet.hr, kozuh@simet.hr, gojic@simet.hr \\ ${ }^{2}$ University of Maribor Faculty of Mechanical Engineering, Maribor, Slovenia, EU \\ ivan.anzel@um.si
}

https://doi.org/10.37904/metal.2019.761

\begin{abstract}
We performed continuous casting of Cu-9Al alloy, following microstructure characterization and computational simulation. Numerical simulation was done on the two scales: within the thermo-mechanical model on the macro scale and the phase-field approach on the mesoscopic scale. In the experimental part of this work, Cu9Al bars were obtained by the continuous casting (CC) process which was subsequently analyzed by optical, scanning electron microscopy, and energy-dispersive X-ray spectroscopy. For the precise quantitative description, a full coupled thermo-mechanical model of the casting process was implemented. Within this model, we successfully numerically simulated time-dependent temperature and solid fraction fields within standard macro phenomenological models. The obtained fields were used as input for a mesoscale multiphase-field model of the constrained dendrite growth into the undercooled melt. Simulations of dendrite structure were compared with the experimental findings and thoroughly analyzed. Two scale simulation frame work has been identified as a useful tool for quantitative prediction of dendrite morphology and CC process optimization.
\end{abstract}

Keywords: Metal processing, continuous casting, solidification, thermo-mechanical, multiphysics

\section{INTRODUCTION}

The rapid advance in understanding the material properties on the fundamental atomistic scale in the middle of the 20th century, together with our knowledge and practical skills enable us to make fast progress in alloy design and production. In this achievement, an interplay of two very different scientific fields, namely physics and metallurgy played a significant role (see the very nice account of the developments by R. Cahn [1]). Our everlasting effort to design, produce, and deploy advanced new materials like shape-memory alloys (SMA) [2] as fast as possible but with reasonable cost poses to us the great challenge of how to achieve it. The complexity of the underlining problem demands new roads to the improvement of every step in the production process. However, the huge progress in the computational techniques make possible to deal with this complexity across the scales. From the materials production point of view, one of the essential technologies is continuous casting (CC). This technology is the first choice whenever we need to obtain long constant crosssection bars economically. One of the significant advantages is that the (semi)product is usually shrinkagefree with stable, functional properties. Although $\mathrm{CC}$ has been used for many years in production lines, especially in the steel industry, we still face many challenges. The essence is how to control the key process parameters, to reach the optimal process conditions for desired microstructure in the final product (see for example [3] and [4]).

Within the available technologies, copper is one of the most important metals, and Cu-based alloys span a huge spectrum of applications. Among others, aluminum bronzes (AB) [5] play an important role because of their high thermal and electrical conductivity accompanied by high corrosion/oxidation resistance. One of the technological off-springs are Cu-based SMAs wherein binary CuAl bases play an important role. The SMA effect shows as more alloying elements are introduced ( $\mathrm{Ni}, \mathrm{Mn}$ are some possibilities [6]). In the field of copper- 
based alloys, including AB, production by $C C$ technology is the Ohno CC system [7] or a horizontal variant of the process [8]. Nevertheless, it is hard to get precise quantitative information about what is going on during solidification and how the microstructure evolves inside the crystalliser. With recent advances in computational technologies and physical models, we are finally able to look into the heart of the CC solidification process by performing numerical experiments on computers.

The material's microstructure (Figure 1) is primarily determined by the solidification process and transformations in the solid-state during cooling. Later, the microstructure can be further modified by thermal and mechanical treatments, and the final microstructure depends on the whole thermomechanical history. For planning the microstructure, the phase-field models (PFM) play a very prominent role. The problem involved consists of the energy scale part that is captured by thermodynamics (Gibbs free energies), the timescale part, which is related to transport (diffusion), and interface introducing length scale. PFM is just convenient physics that allows us to include all phenomena effectively within a consistent mathematical framework [9]. In a recent paper [10] we utilized a simple version of the phase-field model, and here we proceed with a more advanced one that appropriately takes into account solute diffusion and grain orientation in a more appropriate way.
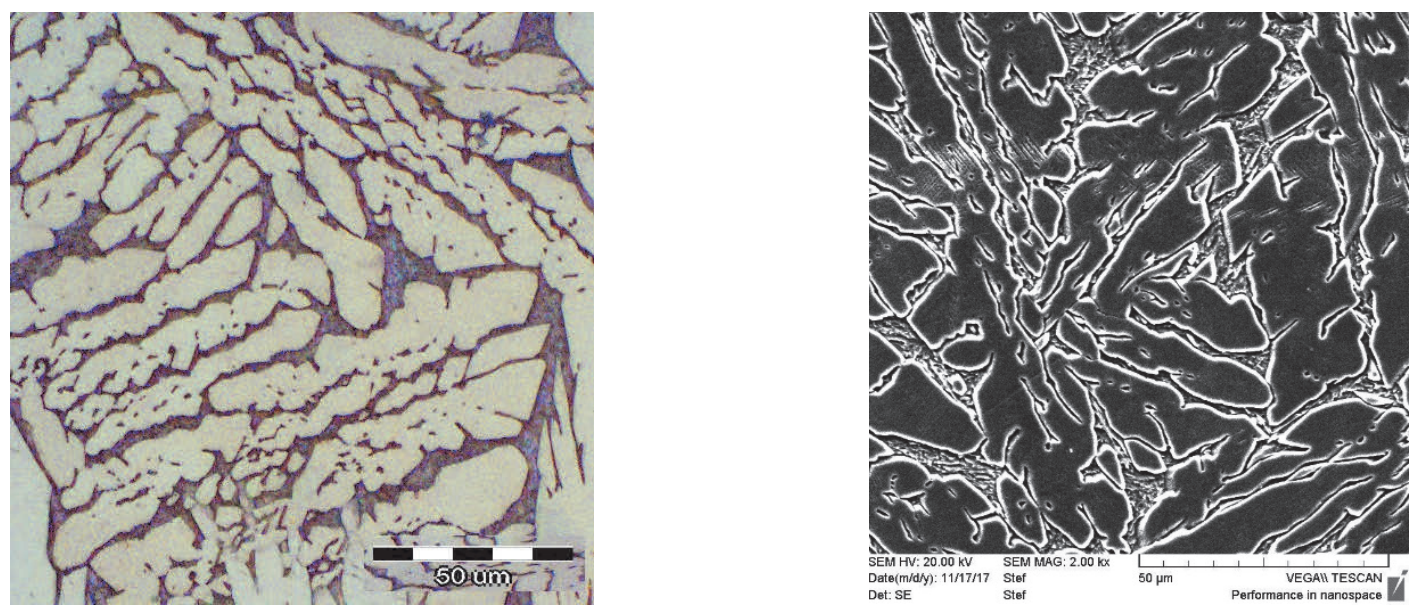

Figure 1 Typical microstructure in the present alloy obtained by microscopy. Positions in (left) and (right) images are approximately similar (due to the measurement process inherent limitations)

\section{EXPERIMENTAL PROCEDURE}

The experimental Cu-9 wt\% Al alloy was prepared by induction vacuum melting using the commercial pure elements (Cu-99.99\%; Al-99.99\%) as starting materials as in [10]. The CC was carried out on a laboratory setup for vertical CC. An adequate quantity of the solid alloy was charged in a graphite crucible and remelted in an induction furnace in a vacuum (1 Pa). After heating up to $1523 \mathrm{~K}$ the melt was continuously cast under an $\mathrm{Ar}$ atmosphere through the graphite mould in which solidification occurs. The withdrawal of the rods was done by the go/stop mode with different go and stop periods. For the successful casting process, it is of utmost importance to properly tune: casting speed, water cooling rate, and liquid alloy temperature in the induction furnace.

Lowering of the cast bar was controlled by the system of rollers that set the casting speed. It was of utmost importance to properly synchronise casting speed with the rate of heat withdrawal in the graphite crystallizer (see the system cross section in Figure 2). If departure from the optimal speed is too high, it leads to solidification in the furnace and too low might result in spillage of the liquid alloy. Even within the functionally correct range, speed is nevertheless the most sensitive process parameter that sets the critical condition for solidification. As demanded by the CC system construction lowering of the cast bar was performed by a regular sequence of $5 \mathrm{~mm}$ pulls with $0.6 \pm 0.1 \mathrm{~s}$ waiting for intervals. As a result, we end up with the average casting 
speed of $26.0 \mathrm{~cm} / \mathrm{min}$. Water cooling system was $10 \mathrm{l} / \mathrm{min}$ providing proper heated withdrawal rate so that we successfully set a stable process condition. Both in the experiment and the thermo-mechanical simulation, it is necessary to let the system achieve a stationary state which means that the conditions of the process and temperature field are constant in time. By this production setup, we obtained dozens of meters long bars $8 \mathrm{~mm}$ in diameter with characteristic surface texture revealing pulls of the transport system.

Figure 1 shows typical optical and SEM micrographs with well-established microstructure in the present system, i.e. a mixture of $\alpha$ and $\beta$ phases. Since the driving force in this solidification process is thermal activation in high-temperature gradients, $\alpha$ phase dominates even though its precipitation might be suppressed since the $\beta$ phase transforms to different phases as a consequence of the violent solute redistribution as cooling goes on [11]. For the microstructure characterization, we have performed optical and scanning electron microscopy accompanied by Energy Dispersive X-Ray Spectroscopy (EDX). The first step was the preparation of the as-cast bar consisting of cutting, polishing, and etching. Thus prepared samples have been examined along with optical and SEM characterisation. The aim was to determine the grains morphology for simulation and experimental results comparison. Figure 1 shows micrographs halfway between the centre and surface of the as-cast bar: (a) optical microscopy, (b) SEM image (2000X magnification) approximately at the same position in the sample as in the optical case. EDX results favour stable chemical composition for the primary phase giving values in the range $6.66-7.58 \mathrm{wt} \%$ of aluminum. Solute concentration distribution confirms that with high cooling rates like in $C \mathrm{C} \beta$-phase transforms into the $\alpha$-phase changing only the crystal structure but keeping the fixed chemical composition. Namely, aluminum bronzes with less than $11 \mathrm{wt} \%$ Al consist primarily of alpha solid solution and some $\beta$-phase (or $\mathrm{k}$ in the presence of $\mathrm{Fe}$ and $\mathrm{Ni}$ alloying elements) that absorbs aluminum from the alpha solid solution reducing overall Al content in $\alpha$-phase. This finding has been confirmed with EDX analysis.

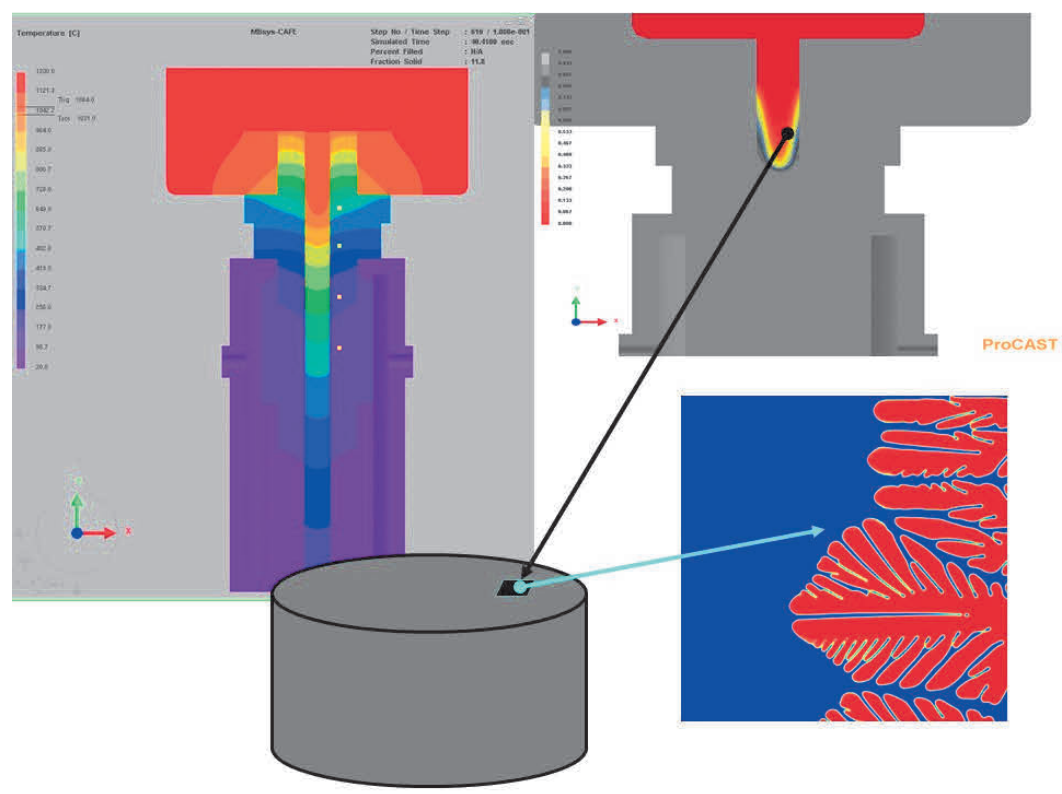

Figure 2 Multiscale simulation ecosystem under stationary conditions. In the left(up) part of the figure, we show the temperature field in the system model with four yellow dots that marks thermometer positions in the experiment. The right part represents solid fraction distribution. Arrows show a tiny piece of the bar position that is modelled by PFM

\section{NUMERICAL SIMULATION}

We performed macroscale simulation by the full non-equilibrium coupled set of thermo-mechanical equations implemented in the ProCAST FE casting simulation software package activating appropriate CC module. Here 
followed a standard simulation strategy by first performing macro heat and mass transport simulation since they govern essential conditions for microstructure evolution during the solidification process. After our CC system achieves stationary condition during casting main macro scale quantity $3 D$ fully resolved thermal gradient was accessible. We have calibrated uncertain parameters of the process (mainly heat transfer coefficients among different materials in the casting system) to faithfully represent thermal gradients in the CC system compared to the measured values. In the simulation, we have used standard values for liquidus $\left(1077^{\circ} \mathrm{C}\right)$, solidus $\left(1040{ }^{\circ} \mathrm{C}\right)$, latent heat $233 \mathrm{~kJ} / \mathrm{kg}$ and density, while specific heat and thermal conductivities are given as functions of temperature. We get physical parameters from standard material databases with some fine tuning to reflect our specific application. Fine-tuning of the process condition parameters (mainly heat transfer coefficient between alloy and graphite crystalliser since it is hard to infer the right values from the experiment due to the high sensitivity on pressure, temperature, and surface texture) ensures that measured values match thermomechanical simulation as in [10]. Casting speed was set to the average value of 26.0 $\mathrm{cm} / \mathrm{min}$ just as in the experiment and is the most sensitive parameter for the real process because it affects heat withdrawal dominantly. Thermal transport, controlled by water cooling, has to be adjusted very precisely to have stable CC process for a long time.

Table 1 Physical material parameters for the process, alloy, and model (no adjustment). Other model parameters are calculated from the ones given here.

\begin{tabular}{|l|c|c|}
\hline Parameter & Symbol & Value \\
\hline Anisotropy coefficient & $\varepsilon_{4}$ & 0.015 \\
\hline Characteristic interface width & $W_{0}$ & $5.0 \times 10^{-7} \mathrm{~m}$ \\
\hline Antitrapping coefficient & $a_{t}$ & 0.35 \\
\hline Liquid diffusion coefficient & $D_{L}$ & $6.5 \times 10^{-9} \mathrm{~m}^{2} / \mathrm{s}$ \\
\hline Melting temperature for Cu & $T_{m}$ & $1358 \mathrm{~K}$ \\
\hline Liquidus temperature & $T_{L}$ & $1350 \mathrm{~K}$ \\
\hline Solidus temperature & $T_{S}$ & $1313 \mathrm{~K}$ \\
\hline Cooling rate & $d q / d t$ & $50 \mathrm{~K} / \mathrm{s}$ \\
\hline Average Al concentration & $C_{\infty}$ & $0.09 \mathrm{Wt} \%$ \\
\hline
\end{tabular}

PFM is one of the most successful approaches in numerical simulation of the microstructure evolution on the mesoscopic scale. The PFM approach encompasses a wide range of physical processes, most notably solidification phenomena. There exists extensive literature on the subject, and the interested reader is referred to the comprehensive review papers devoted to the subject (see for example [9] or [12]). One recent development is a possibility to take into account the motion of growing dendrites [13] phenomenon which was consistently neglected in the past. The model utilized here is based on the non-variation version of the multiPFM by N. Ofori-Opoku and N. Provatas [14]. The system of partial differential equations for different orientations phase-fields $\phi_{i}$ and solute concentration $c$ are given by equations 35 and 36 from [14]. Here we strictly follow procedure and symbol conventions of that paper and omit them here because of the relative complexity.

The model itself is deeply nonlinear and numerically demanding. It is solved using a finite volume scheme where an OpenMP parallel method for improved efficiency on multi-core CPU architecture has also been employed. One of the features of this PFM is efficient elimination of the spurious kinetics by the addition of the anti-trapping flux term, the technique proposed in [15] by A. Karma and co-workers.

Figure 2 shows the general scheme in this simulation. We start from the macro environment set by thermomechanical model results like in [10] and impose them as a thermal environment for PFM simulation. For the simulation domain, we take a 2D square (Figure 3 ) that represents a tiny part of the horizontal cross-section 
that lowers down together with the casting bar. Simulation cell corresponds to the micrograph shown in Figure 1 on the right (SEM). Thus we simulate microstructure evolution as seen down the casting bar, and grain growth directed toward the bar symmetry axis in the horizontal plane. Growth direction is a consequence of the macro thermal gradients and thus heat transport directions in the present CC system. It is essential to include heat transport along the casting bar downwards since this route also contributes to the heat withdrawal. We solve PFM equations in the frame of reference set by the speed of the moving bar. Our simulation domain is, therefore, going downward at the same speed as the bar itself looking from the LAB frame of reference. It means that temperature is decreasing simultaneously in every point of the simulation domain. The space temperature gradient along the vertical direction is present here as the time rate of heat withdrawal $\mathrm{dq} / \mathrm{dt}$. In Figure $\mathbf{3}$ we show the main result of this research that corresponds to the micrographs in Figure 1. Microstructure in Figure 3 is the numerical simulation result with physical and process parameters given in Table 1. The main influence on the solidification and dendrite morphology comes from relatively rapid cooling as the simulation domain lowers down.
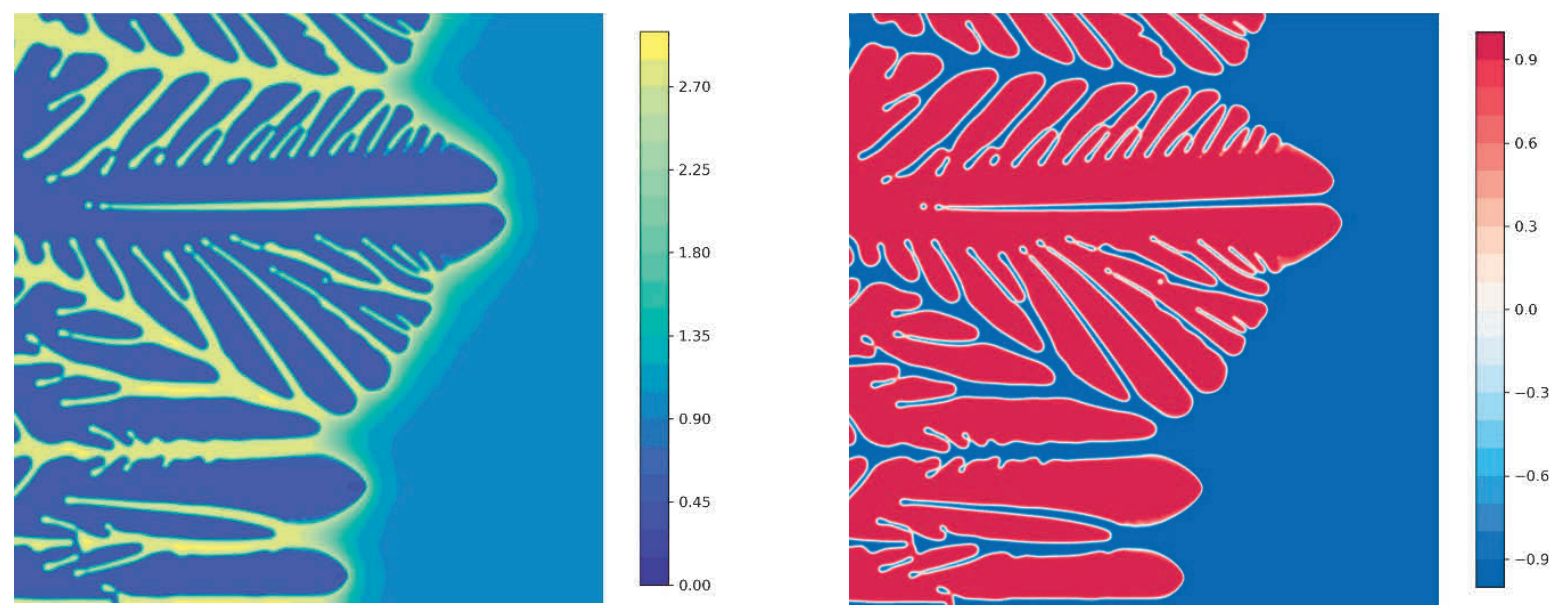

Figure 3 Dendritic microstructure evolved in the PFM simulation with a characteristic pattern for the present cooling rate. In the left figure, we show solute concentration and in the right figure sum of the phase-fields for each grain. The simulation cell was quadratic with dimensions of $50 \times 50 \mu \mathrm{m}$

In numerical simulations, we have used process parameters given in Table 1. The system has been cooled at the rate given by $d q / d t$ that governs partially directional character of the solidification process. Our numerical calculations give good agreement with the experimental value of the solute concentration within the primary phase that is around $7 \mathrm{wt} \%$, and full distribution is shown in Figure 3. The experimental finding by EDX spectroscopy is in the range [7-8] wt\% giving us confidence that computational model captures main solute redistribution trend in this $\mathrm{CC}$ solidification process and that a current antitrapping scheme as proposed by Karma [15] works well in our case. Besides qualitative dendrite morphology correspondence, dendrite arm doubling and spacings (around $10 \mu \mathrm{m}$ ) are in good agreement. However, we note that the model is unable to properly describe grain coalescence and give quantitative agreement for secondary dendrite arms spacing.

\section{CONCLUSION}

In conclusion, we have carried out the experimental and computational study of the solidification process for binary Cu-9Al alloy. For the experimental characterisation, we did standard optical microscopy and SEM accompanied by EDX analysis. For the computational simulation, we proceed using a specially adapted version of the multi PFM [14] for the solidification and industry standard suite ProCAST for the thermomechanical processes scale simulation. Micrographs obtained by microscopy compare favourably with PFM simulated microstructure and show quantitative agreement for solute microsegregation. We emphasise that 
we used a standard process and material parameter practically without values adjustments giving us confidence about the predictive strength of the present simulation approach. One obvious improvement of the present model could be coupling to heat diffusion together with latent heat release on the solidification front. This issue needs to be addressed in future research involving an estimate of the benefit contrasted to increased model complexity. To conclude we presented full macro and mesoscale model for CC of Cu-9AI bars where we can numerically simulate relevant microstructural features: solute redistribution and dendrite arms spacing. Quantitative accuracy gives us confidence in the method and paves a direction for practical improvement of our CC process. Since the scheme is quite general, we expect a successful application for optimal simulation results also in different alloys relevant for industrial applications.

\section{ACKNOWLEDGEMENTS}

This work was supported by the Croatian Science Foundation under Grant (IP-2014-09-3405).

\section{REFERENCES}

[1] CAHN, R.W. Has the child of metallurgy walked out on its parent? MRS Bulletin. 2003. vol. 28, no. 7, pp. 468-469.

[2] WAYMAN, C.M. and OTSUKA, K. editors. Shape memory materials. 1st ed.: Cambridge University Press; 1999.

[3] THOMAS, B.G. Review on Modelling and Simulation of Continuous Casting. Steel Research Int. 2017. vol. 89, no. 1, pp. 1700312.

[4] VERTNIK, R. and ŠARLER, B. Simulation of continuous casting of steel by a meshless technique. International Journal of Cast Metals Research. 2009. vol. 22, no. 1-4, pp. 311-313.

[5] MEIGH, H. Cast and wrought aluminium bronzes: Properties, processes and structure. 1st ed. London: CRC Press; 2000. Engineering \& Technology, Physical Sciences.

[6] GOJIĆ, M., VRSALOVIĆ, L., KOŽUH, S. et al. Electrochemical and microstructural study of Cu-Al-Ni shape memory alloy. Journal of Alloys and Compounds. 2011. vol. 509, no. 41, pp. 9782- 9790.

[7] OKAYASU, M., TAKASU, S. and YOSHIE, S. Microstructure and material properties of an Al-Cu alloy provided by the Ohno continuous casting technique. Journal of Materials Processing Technology. 2010. vol. 210, no. 11, pp. 1529 - 1535.

[8] MAHMOUDI, J. Horizontal continuous casting of copper-based alloys. International Journal of Cast Metals Research. 2005. vol. 18, no. 6, pp. 355-369.

[9] STEINBACH, I. Phase-field models in materials science. Modelling and Simulation in Materials Science and Engineering. 2009. vol. 17, no. 7, pp. 073001.

[10] PEZER, R., KOŽUH, S., ANŽEL, I. et al. Optimizations in continuous casting of Cu based alloys by numerical simulation. In METAL 2018: 27th International Conference on Metallurgy and Materials. Ostrava: TANGER, 2018. pp. 1407 - 1414.

[11] CENOZ, I. and GUTIERREZ, M. Phase transformations in Cu - Al Alloy. Metal Science and Heat Treatment 2011, vol. 53, no. 5/6, pp. $265-269$.

[12] PROVATAS, N., GREENWOOD. M., ATHREYA, B. et al. Multiscale modelling of solidification: Phase field methods to adaptive mesh refinement. International Journal of Modern Physics B. 2005. vol. 19, no. 31, pp. 45254565 .

[13] QI, X.B., CHEN, Y., KANG, X.H. et al. Modelling of coupled motion and growth interaction of equiaxed dendritic crystals in a binary alloy during solidification. Scientific Reports. 2017. vol. 7, pp. 45770.

[14] OFORI-OPOKU, N., PROVATAS, N. A quantitative multi-phase field model of polycrystalline alloy solidification. Acta Materialia. 2010. vol. 58, no. 6, pp. 2155 - 2164.

[15] KARMA, A. Phase-field formulation for quantitative modelling of alloy solidification. Phys Rev Lett. 2001. vol. 87, pp. 115701.

[16] ECHEBARRIA, B., FOLCH, R., KARMA, A. et al. Quantitative phase-field model of alloy solidification. Phys Rev E. 2004. vol. 70, pp. 061604. 